\title{
Objective-Prism Emission-Line Searches for Active Galactic Nuclei
}

\author{
Caryl Gronwall \\ Dept. of Physics 6 S Astronomy, Johns Hopkins University, Baltimore \\ $M D 21218$ \\ Vicki L. Sarajedini \\ Dept. of Astronomy, University of Florida, Gainesville, FL 32611
}

\author{
John J. Salzer \\ Astronomy Dept., Wesleyan University, Middletown, CT 06459
}

\begin{abstract}
Objective-prism surveys for UV-excess and emission-line objects, especially the First and Second Byurakan Surveys, have been central to the study of active galactic nuclei (AGNs). We review previous line-selected surveys for AGNs and discuss their contribution to our understanding of the AGN phenomena. In addition, we present results from the KPNO International Spectroscopic Survey, a modern digital objective-prism survey for emission line objects. This survey is discovering substantial numbers of new AGNs, in particular low-luminosity AGNs and LINERs.
\end{abstract}

\section{Introduction}

Objective-prism surveys revolutionized our knowledge of active galactic nuclei (AGNs) in the local universe. In particular, many of the known nearby Seyfert galaxies were discovered by Markarian in the First Byurakan Survey (FBS; see Khachikian this volume.) Galaxies were selected for inclusion in the FBS based on the presence of a UV excess. This selection criterion is biased against the discovery of more heavily reddened AGN such as Seyfert 2's and LINER galaxies. A number of line-selected surveys have been carried out over the past 20 years in order to select well-defined samples of local AGNs. In this paper, we review existing line-selected surveys for local AGNs carried out using objective-prism techniques (see Stepanian, this volume, for a discussion of the UV+line selected Second Byurakan Survey). We then present a discussion of a new, modern digital objective-prism survey for emission-line galaxies (ELGs), the KPNO International Spectroscopic Survey (KISS). KISS probes several magnitudes deeper than existing surveys, and due to its selection via $\mathrm{H} \alpha$ emission, is less biased against Seyfert 2 and LINER galaxies than previous surveys. KISS provides one of the best, well-defined samples of local AGNs for comparison to higher redshift studies. 


\section{Previous Line-Selected Surveys}

The use of line-selected objective-prism surveys to detect active and star-forming galaxies in the local universe has a long and fruitful history (e.g., Smith 1975; Smith et al. 1976). The University of Michigan (UM) survey (MacAlpine et al. 1977; MacAlpine \& Lewis 1981) selected objects via their [OIII] $\lambda 5007$ emission line. 349 ELGs were detected in $667 \mathrm{sq}$. degrees. This is a number density of 0.52 per square degree. In Lists $4 \& 5$ of the UM survey (for which complete follow-up spectroscopy is available from Salzer et al. 1989) 166 ELGs are found in $325 \mathrm{sq}$. degrees. Approximately $10 \%$ of the ELGs detected in the UM survey are AGNs: 9 Seyfert 1's and 7 Seyfert 2's. Wasilewski (1983) also utilized the [OIII]-line as his selection criterion and found 96 ELGs in 825 sq. degrees, or 0.18 per square degree. Wasilewski found that $8 \%$ are AGNs with 1 Seyfert 1 and 7 Seyfert 2 galaxies being detected. The Case survey (Pesch \& Sanduleak 1983; Stephenson et al. 1992) used two selection criteria, both UV-excess and [OIII]-line emission and cataloged 1440 ELGs in 1551 sq. degrees or 0.94 per sq. degree. Salzer et al. (1995) obtained follow-up spectroscopy for a complete subsample of 176 of the Case galaxies and found that only $6 \%$ are AGNs: 2 Seyfert 1's, 7 Seyfert 2's, and 2 LINERs. Most recently the Universidad Complutense de Madrid (UCM; Zamorano et al. 1994, 1996) survey utilized selection via $\mathrm{H} \alpha$-emission to discover 263 ELGs in 471 sq. degrees or 0.56 per square degree. Follow-up spectroscopy by Gallego et al. (1997) found that 14 (or $5 \%$ ) of these are AGNs, including 5 Seyfert 1's and 9 Seyfert 2's. The completeness limits of all of the above surveys range from $B=15$ to 17 .

Most of the previous line-selected surveys discussed above have been done in the blue (selecting via [OIII $] \lambda 5007$ emission) and all used photographic plates. There are a number of biases introduced by these methods. First, selection in the blue limits the sensitivity of the surveys to more heavily reddened Seyfert 2's and low-ionization LINER galaxies. Second, because the standard IIIa-J emulsions used in most photographic surveys cut off at $\sim 5350 \AA$, the redshift depth of these surveys is limited to $z \sim 0.065$. A modern, digital survey using CCDs to substantially improve survey depth (to fainter magnitudes and higher redshifts) plus selecting via a redder line $(\mathrm{H} \alpha)$ would greatly reduce these biases. Such a survey is now available, the KPNO International Spectroscopic Survey (KISS).

\section{The KPNO International Spectroscopic Survey}

The KPNO International Spectroscopic Survey (KISS) was initiated in 1994 as a collaborative effort between astronomers from Russia, Ukraine, and the US who shared a common interest in the study of galaxian activity. The survey was envisioned as being the "next generation Markarian survey" for active and star-forming galaxies. By combining the traditional objective-prism survey method for emission line detection with modern detector technology and computer-based analysis, KISS is able to discover Seyfert Galaxies (Sy 1/Sy 2), LINER's, Starburst Nucleus Galaxies (SBNs), HII Galaxies, and Blue Compact Dwarfs (BCDs) at least 2 magnitudes fainter than previous work. The survey is thus a unique resource for probing the nature of star-forming and active galaxies 
in the nearby universe. A full description of the survey can be found in Salzer et al. $(2000,2001)$.

The KISS survey is conducted with CCD detectors on the 24-inch Burrell Schmidt on Kitt Peak. For the first survey strip, a $2048^{2}$ CCD was used, which afforded a field-of-view of 70 arcmin $\left(1.18^{\circ}\right)$ square, with a scale of 2.03 arcsec/pixel. This one-degree-wide strip was chosen to coincide with the Century Redshift survey (Geller et al. 1997) covering a strip at a constant declination of $29^{\circ}$ from $\mathrm{RA}=12^{h} 15^{m}$ to $17^{h}$ which encompasses an area of $62 \mathrm{deg}^{2}$. The second survey strip goes through the Boötes void at a constant declination of $43^{\circ}$ from $\mathrm{RA}=12^{h}$ to $16^{h} 15^{m}$ covering an area of $66 \mathrm{deg}{ }^{2}$. For the second strip a $2048 \times 4096$ CCD with $1.45 \mathrm{arcsec} /$ pixel resolution was used. The KISS survey data consists of broadband $B$ and $V$ images (for astrometric and photometric calibration), and spectral (objective prism) data. The spectra were taken in the red (using a blocking filter to restrict the wavelength range to 6400-7200 $\AA$ ) to detect $\mathrm{H} \alpha$ emission. One of the important features of the KISS project is its objective criteria: the search and measurement process is carried out entirely by computer. A complete package of IRAF scripts and executables analyzes every source in the field (typically 5000 - 7000 objects) automatically; the output of these routines is a catalog of photometric and astrometric data, and an extracted spectrum for every object in the field. In addition, a list of ELG candidates is produced.

Our survey technique has proven to be quite successful. In our first red spectral strip we have cataloged 1128 ELG candidates. This is about $18.1 \mathrm{per}$ square degree. In the second survey strip, we have detected 1030 ELG candidates or 15.6 per square degree. For comparison, the entire Markarian survey cataloged 1500 UV-excess galaxies (0.1 galaxy per square degree), and had a completeness limit of $\mathrm{m}_{B}=15.2$ (Mazzarella \& Balzano 1986), while the deeper UM (median $\mathrm{m}_{B}=16.9$; MacAlpine et al. 1977) and UCM (median $\mathrm{m}_{B}=16.5$; Zamarano et al. 1994) surveys both detected only 0.5 ELGs per square degree.

\subsection{Spectroscopic Follow-up}

We have obtained follow-up spectroscopy for 725 of 1128 ELG candidates selected via $\mathrm{H} \alpha$ emission from the first survey strip using the WIYN $3.5-\mathrm{m}$, the KPNO 2.1-m, the MDM 2.4-m, the APO 3.5-m, and the Lick 3-m telescopes as well as the Hobby Eberly Telescope. These spectra provide redshifts, $\mathrm{H} \alpha$ equivalent widths, and line fluxes for various important emission lines including $\mathrm{H} \beta$, [O III] $\lambda \lambda 4959,5007, \mathrm{HeI} \lambda 5876$, [O I] $\lambda 6300, \mathrm{H} \alpha,[\mathrm{N}$ II] $\lambda \lambda 6548,6583$, and [S II] $\lambda \lambda 6717,6731$. Measurements of the Balmer decrement allow us to directly measure the extinction $\left(A_{V}\right)$ for each of these galaxies. The follow-up spectra also allow us to identify AGNs in our sample: Seyfert 1s are identified via their broad permitted emission lines, while Seyfert 2's and LINERs are distinguished primarily via the $[\mathrm{OIII}] / \mathrm{H} \beta$ line ratio with LINERs having $[\mathrm{OIII}] / \mathrm{H} \beta<3$ (e.g., Veilleux \& Osterbrock 1987). Representative spectra of AGN in the KISS sample are shown in Figures $1 \& 2$.

We find that $91 \%$ (663) of the KISS candidate ELGs are confirmed emissionlines galaxies. About 11\% of the galaxies are active galactic nuclei with 67 (10 Seyfert 1's, 19 Seyfert 2's, and 38 LINER galaxies) detected via $\mathrm{H} \alpha$ with redshifts less than 0.095, and an additional 10 (1 Seyfert 1, 5 Seyfert 2's and 4 QSO's) 


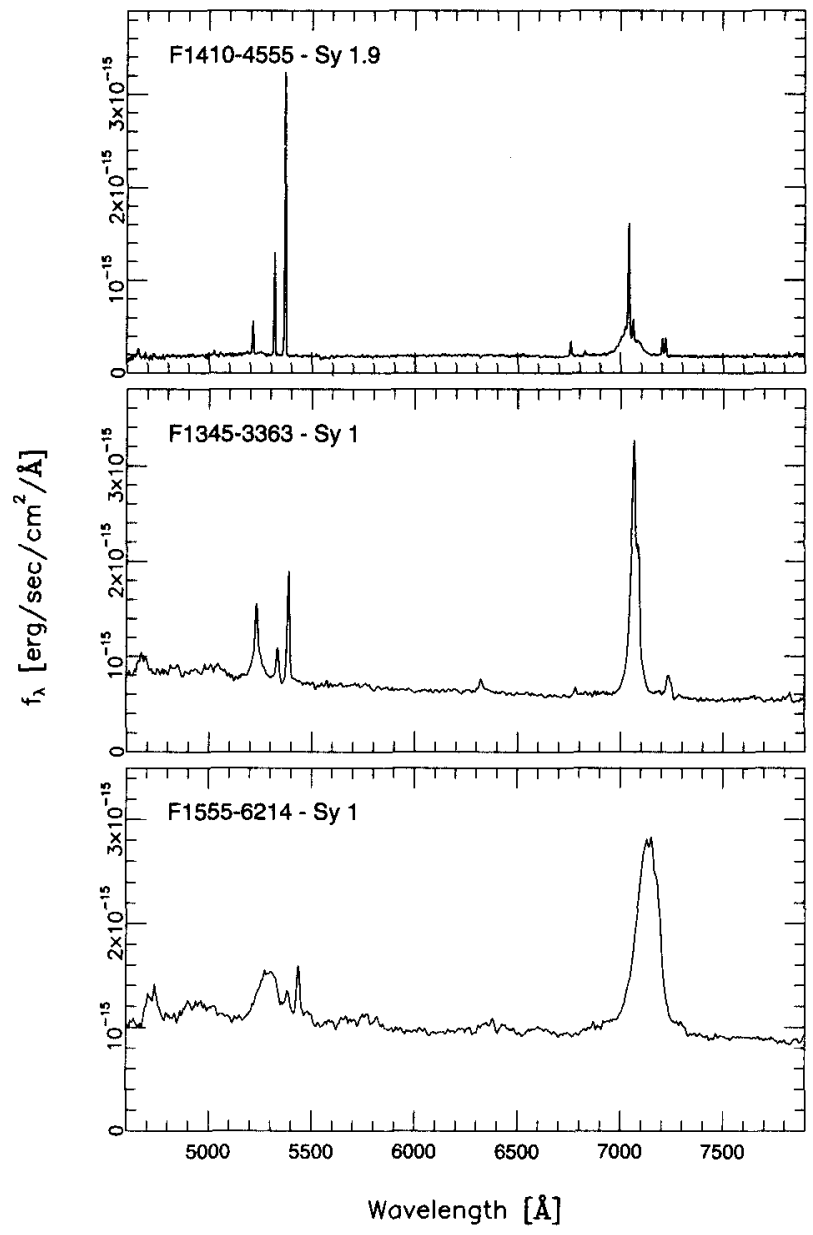

Figure 1. Representative spectra of KISS Seyfert 1 galaxies. 


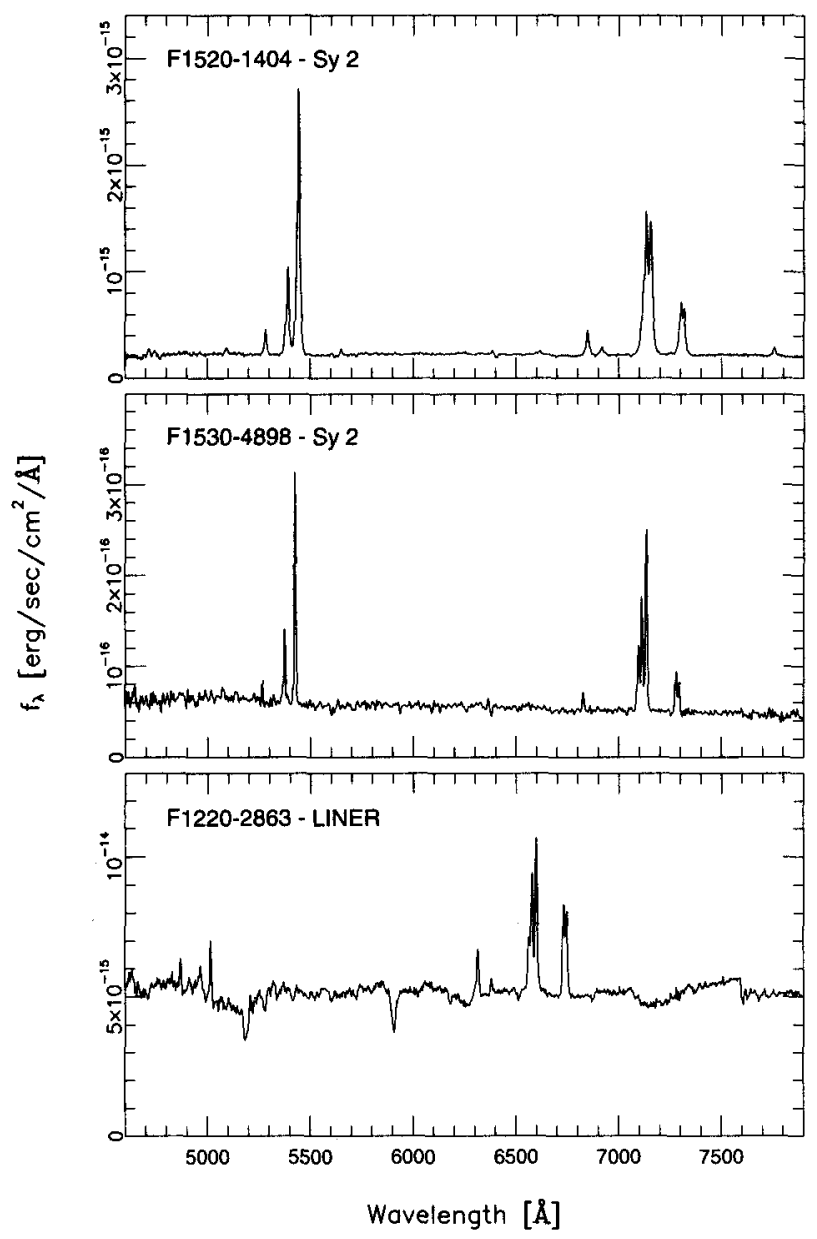

Figure 2. Representative spectra of KISS Seyfert 2 and LINER galaxies. 


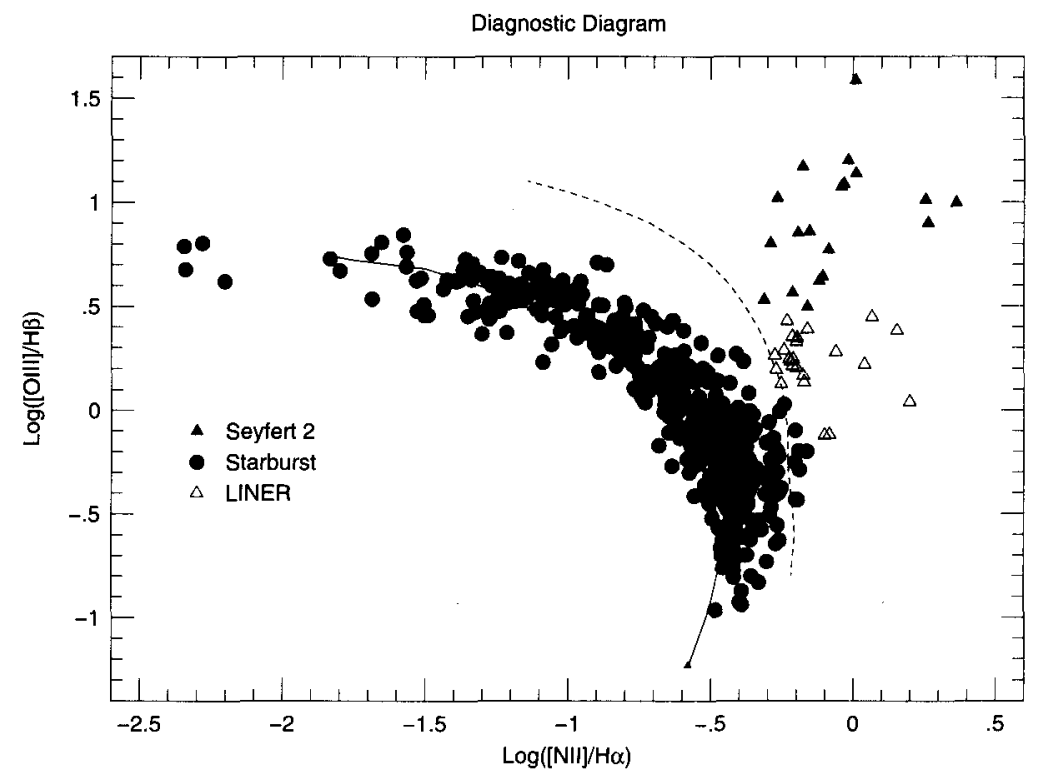

Figure 3. Line diagnostic diagram plotting the logarithm of $[\mathrm{O}$ III $] \lambda 5007 / \mathrm{H} \beta$ against the logarithm of [N II] $\lambda 6583 / \mathrm{H} \alpha$. Solid circles represent star-forming ELGs, open triangles are LINERS, and the solid triangles are Seyfert 2 galaxies. The solid line represents an HII model sequence at various metallicities from $0.1 \mathrm{Z}_{\odot}$ at the upper left to $2 \mathrm{Z}_{\odot}$ at the lower right (Dopita \& Evans 1986).

detected at higher redshifts where another line has redshifted into our filter. A line diagnostic diagram plotting the logarithm of $[\mathrm{O}$ III $] \lambda 5007 / \mathrm{H} \beta$ against the logarithm of [N II] $\lambda 6583 / \mathrm{H} \alpha$ is shown in Figure 3. The solid line represents the HII sequence with low metallicity, high-ionization ELGs in the upper left and high metallicity, low-ionization objects in the lower right part of the sequence. The star-forming ELGs follow the HII sequence allowing us to classify them from BCDs in the upper left to starburst nuclei in the lower right. AGNs are clearly separated from star-forming galaxies. We find many more high metallicity, lowionization objects than found with traditional [O III] $\lambda 5007$-selected surveys. The data make it clear that $\mathrm{H} \alpha$-selected surveys are much more effective at detecting the full range of ionization and metallicity present in star-forming galaxies than [O III] or [O II] observations.

\subsection{Properties of KISS AGN}

We will concentrate here on the $67 \mathrm{H} \alpha$-selected AGN which constitute a welldefined local sample of AGN. Note that because our survey has a sharp upper- 


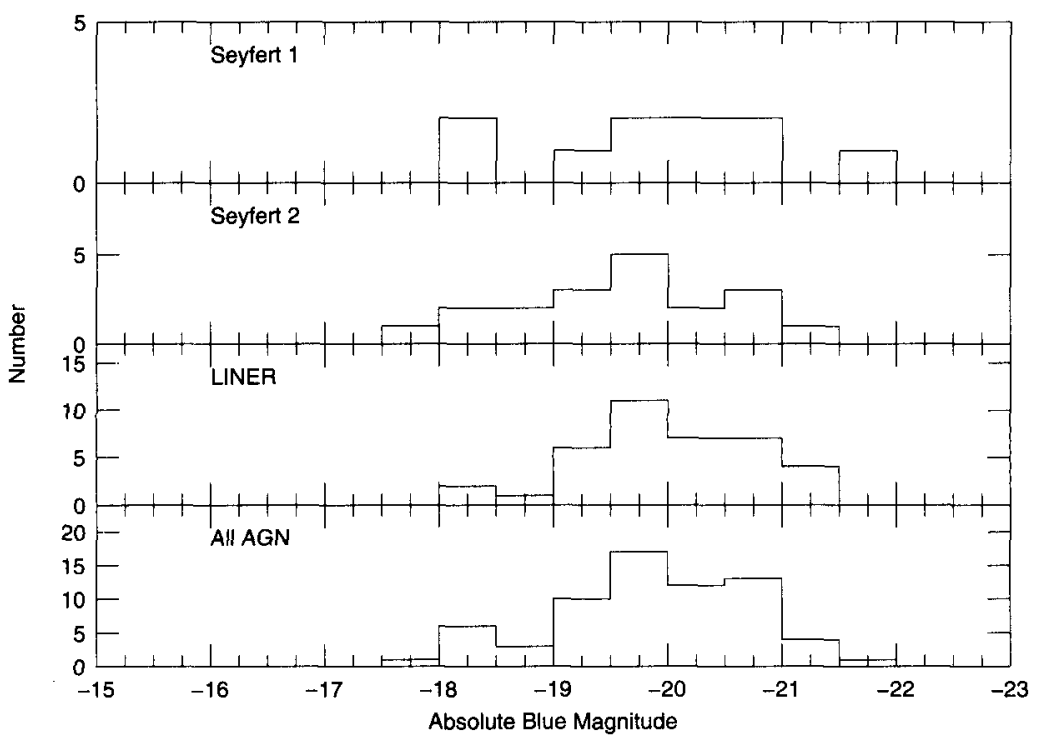

Figure 4. $B-V$ colors of Seyfert 1's, Seyfert 2's, LINER's and all KISS AGN.

wavelength cutoff due to the blocking filter used, the sample is volume-limited for the more luminous objects and all but 8 of the AGNs fall in this category. Figure 4 shows the integrated absolute magnitude distributions of the AGNs in the local KISS sample. KISS detects AGNs with $M_{B}$ ranging from -21.8 to -18 (assuming $H_{0}=75 \mathrm{~km} \mathrm{~s}^{-1} \mathrm{Mpc}^{-1}$ ). The Seyfert 1, Seyfert 2, and LINER luminosity distributions look similar. Figure 5 shows the $B-V$ color distributions of the KISS AGNs. As expected, Seyfert 2 and LINER distributions are significantly redder (median $B-V=0.92$ and 0.93 respectively) than the Seyfert 1 color distribution (median $B-V=0.70$ ). Because of their redder colors, an $\mathrm{H} \alpha-$ selected survey is much more sensitive to Seyfert 2's and LINERs than a blue (e.g., UV excess or [OIII]-selected) survey. This is borne out by comparing the 2:1 LINER/Sy 2 ratio in the KISS survey to the complete lack of LINERs discovered in the [OIII]-selected surveys discussed in Section 2.

We have also calculated the $B$-band luminosity function for the Seyfert galaxies in our sample. Because our direct images lack the angular resolution required to resolve the nucleus, this is an integrated luminosity function. However, it is directly comparable to the integrated local Seyfert luminosity function of Huchra \& Burg (1992). Figure 6 shows the blue luminosity function for both the KISS sample and that of Huchra \& Burg. The two LFs are consistent, with the KISS LF extending to fainter luminosities. Note that for this calculation we 


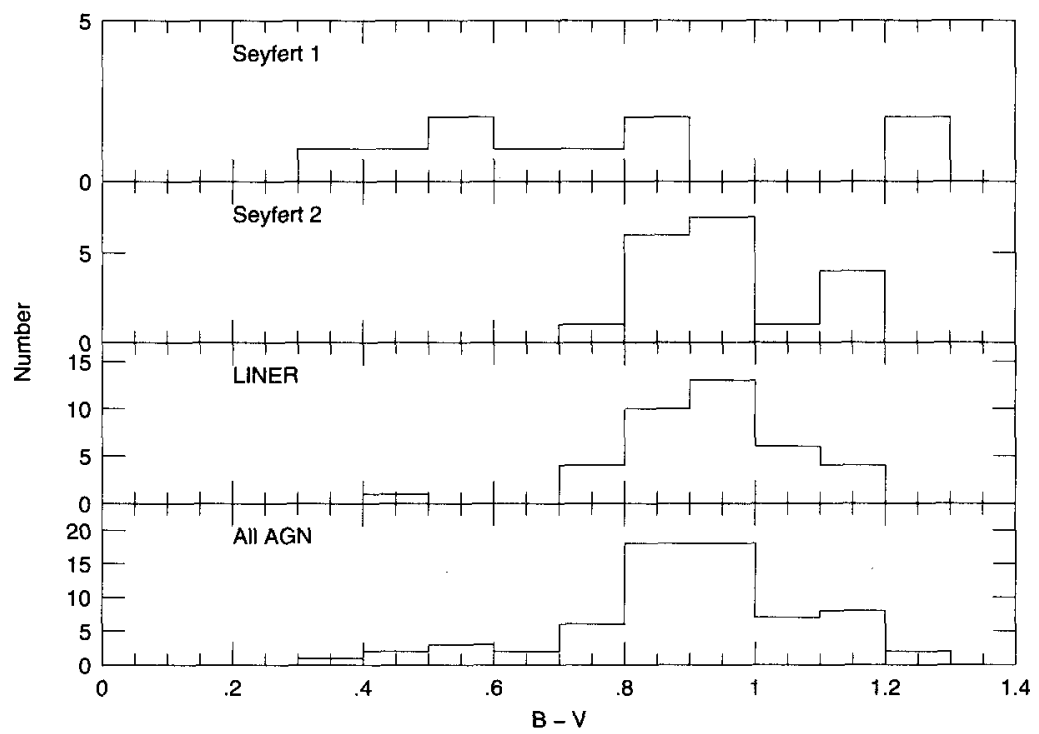

Figure 5. $\quad B-V$ colors of Seyfert 1's, Seyfert 2's, LINER's and all KISS AGN.

have not accounted for the possibility that there are AGNs in the fraction of our sample for which we have no follow-up spectra. Because we have preferentially obtained spectra for the more luminous galaxies in our sample, we expect that such a correction would be small, but it could increase our volume densities slightly. Further discussion of the LF and its implications for AGN evolution when compared to other local and higher redshift AGN LFs will be discussed in a future paper (Sarajedini, Gronwall \& Salzer 2002).

\subsection{Multiwavelength Properties}

We have also cross-correlated our $\mathrm{H} \alpha$-selected sample of ELGs from both our first and second survey strips with surveys done in the x-ray, radio, and the far-infrared. A comparison of the KISS sample with the ROSAT All-sky Survey (Voges et al. 1999) finds that 17 of the 2158 KISS ELGs are detected by ROSAT. These are all AGNs: 13 Sy 1's, 2 Sy 2's, and 2 LINERs. The x-ray detected AGNs are primarily the most luminous AGNs detected by KISS. A cross-correlation of the KISS sample with the $1.4 \mathrm{GHz}$ FIRST survey (White et al. 1997) done with the VLA reveals that 178 of the 2158 KISS ELGs are detected by FIRST. We have follow-up spectra for all of these sources and find 5 Sy 1's, 28 Sy 2's, and 30 LINERs. Similarly, 154 of the 2158 KISS ELGs are found in the IRAS Faint Source Catalog (Moshir et al. 1992) and follow-spectra for these sources 
Seyfert 1+2 Luminosity Function

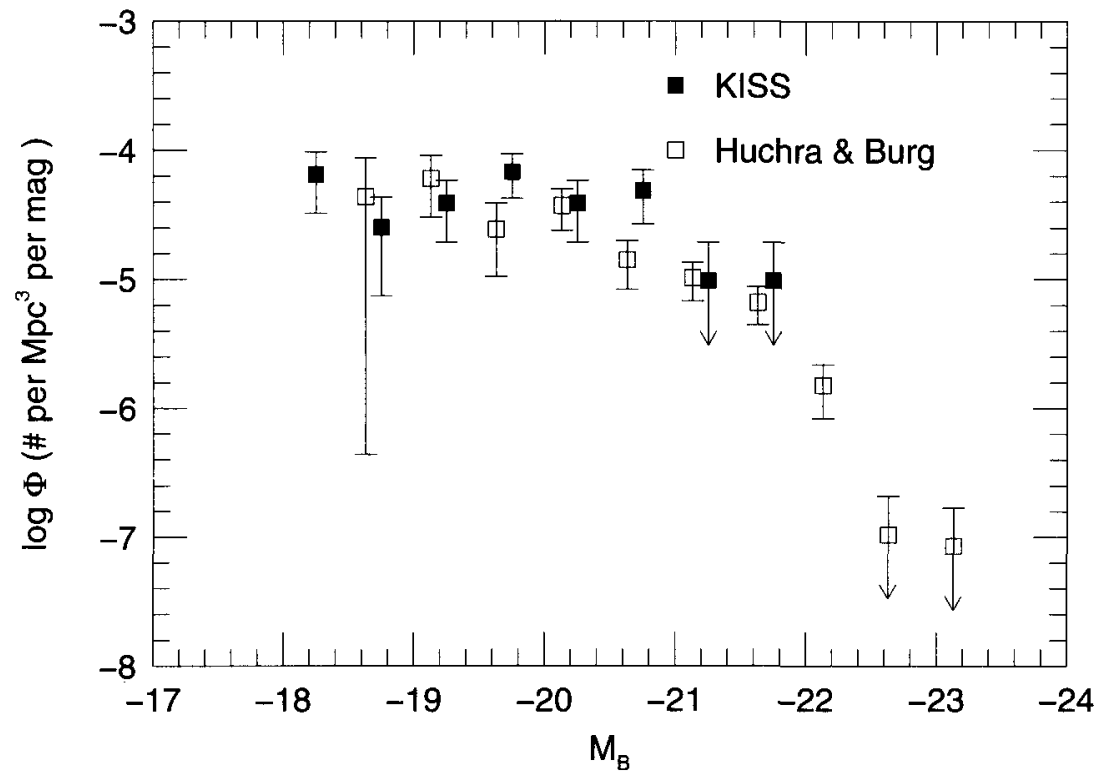

Figure 6. Integrated blue luminosity function for Seyfert galaxies in the KISS AGN sample. The KISS luminosity function is shown in solid squares while the luminosity function from Huchra \& Burg (1992) is shown with open squares.

have detected 1 Sy 1, 9 Sy 2's, and 12 LINERs. The KISS ELGs follow the wellknown radio-IR correlation discovered by Condon et al. (1991). This correlation is thought to be due to the fact that both the radio and far-IR emission are dominated by star formation. Somewhat surprisingly, we find that the majority of the AGNs in our sample follow the same radio-IR correlation as the starforming galaxies, implying that the dominant ionizing source in these objects might be young stars, not the active nucleus. We are currently investigating this possiblity. We also plan to use these data to test the validity of IR color diagnostics for AGN activity.

\section{Summary}

The KISS sample represents a substantial step forward in our understanding of the AGN population in the local universe. It is essentially volume-limited and less biased against redder Seyfert 2 and LINER galaxies, and provides a well-defined sample of nearby $(z<0.1)$ AGN with which to study the statistical properties of AGN. In the first KISS survey strip covering 62 square degrees, 10 
Seyfert 1's, 19 Seyfert 2's, and 38 LINER's were discovered. KISS provides an essential database of local AGNs for comparison to higher redshift samples.

Acknowledgments. CG is grateful to the American Astronomical Society and the International Astronomical Union for travel grants which allowed her to participate in this meeting. She would also like to thank the Local Organizing Committee for their wonderful hospitality.

\section{References}

Condon, J.J., Anderson, M.L., \& Helou, G. 1991, ApJ, 376, 95

Dopita, M.A., \& Evans, I.N. 1986, ApJ, 307, 431

Gallego, J., Zamorano, J., Rego, M., \& Vitores, A. G. 1997, ApJ, 475, 502

Geller, M. J. et al. 1997, AJ, 114, 2205

Huchra, J. \& Burg, R. 1992, ApJ, 393, 90

Khachikian, E. Ye. 2001, this volume

MacAlpine, G. M., Smith, S. B., \& Lewis, D. W. 1977, ApJS, 34, 95

MacAlpine, G. M., \& Williams, G. A. 1981, ApJS, 34, 95

Mazzarella, J. M., \& Balzano, V. A. 1986, ApJS, 62, 751

Moshir, M. et al. 1992, Explanatory Supplement to the IRAS Faint Source Survey, Version 2, JPL D-10015, (Pasadena: JPL)

Pesch, P. \& Sanduleak, N. 1983, ApJS, 51, 171

Salzer, J. J., MacAlpine, G. M., \& Boroson, T. A. 1989, ApJS, 70, 479

Salzer, J. J., Moody, J. W., Rosenberg, J. L., Gregory, S. A., \& Newberry, M. V. 1995, AJ, 109, 2376

Salzer, J. J., Gronwall, C., Lipovetsky, V. A., Kniazev, A., Moody, J. W., Boroson, T. A., Thuan, T. X., Izotov, Y. I., Herrero, J. L., \& Frattare, L. M. 2000, AJ, 120, 80

Salzer, J. J., Gronwall, C., Lipovetsky, V. A., Kniazev, A., Moody, J. W., Boroson, T. A., Thuan, T. X., Izotov, Y. I., Herrero, J. L., \& Frattare, L. M. 2001, AJ, 121, 66

Sanduleak, N. \& Pesch, P. 1984, ApJS, 55, 517

Sarajedini, V.L., Gronwall, C., \& Salzer, J.J. 2002, in preparation

Smith, M. G. 1975, ApJ, 202, 591

Smith, M. G., Aguirre, C., \& Zemelman, M. 1976, ApJS, 32, 217

Stepanian, J.A. 2001, this volume

Stephenson, C. B., Pesch, P., \& MacConnell, D. J. 1992, ApJS, 32, 217

Veilleux, S. \& Osterbrock, D. E. 1987, ApJS, 63, 295

Voges, W. et al. 1999, A\&A, 349, 389

Wasilewski, A.J. 1983, ApJ, 272, 68

White, R. L., Becker, R. H., Helfand, D. J., \& Gregg, M. D. 1997, ApJ, 475, 479

Zamorano, J., et al. 1996, ApJS, 104, 99

Zamorano, J., et al. 1994, ApJS, 95, 387 